\title{
Early Unfavorable Hodgkin Lymphoma
}

National Cancer Institute

\section{Source}

National Cancer Institute. Early Unfavorable Hodgkin Lymphoma. NCI Thesaurus. Code C68664.

An early Hodg kin lymphoma with unfavorable prognosis. 